\title{
COVID-19: La necesidad de un control más estricto sobre el alcohol no registrado en Rusia
}

\section{COVID-19: A need for stricter control over unrecorded alcohol in Russia}

\author{
Artyom GiL*. \\ * Institute for Leadership and Health Management, I.M. Sechenov First Moscow State Medical University. \\ Sechenov University, Moscú. Rusia.
}

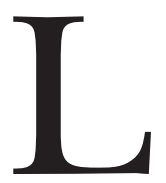

a pandemia de la COVID-19 ha impactado de manera significativa aspectos de la vida y afectado directa o indirectamente la salud de la población en muchos países (Lancet COVID-19 Commissioners et al., 2020; OMS, 2020). En Rusia, entre enero y agosto 2020, el número total de muertes por cada 100000 habitantes aumentó por 0,8 casos en comparación con el mismo período de 2019 (13,2/100 000 en 2020 vs. 12,4/100 000 habitantes en 2019). La tasa de desempleo registrada aumentó en un 2,1\% y representó el 6,4\%. En el segundo trimestre de 2020, cuando se introdujo un régimen de «autoaislamiento" para frenar la propagación de la infección por SARS-CoV-2, la renta real disponible de la población disminuyó en un 8,4 \% en comparación con el mismo período en 2019 (RosStat, 2020). El $61 \%$ de la población de Rusia observó una disminución en los ingresos, el 13,5 \% reportó una pérdida total de los ingresos, mientras que el 9,8\% perdió su empleo por completo (Artamonov y Lavrentyev, 2020).

Simultáneamente con estos cambios, durante los primeros meses de la pandemia de SARS-CoV-2, varias regiones de la Federación Rusa (Karelia, Yakutia, Tuva, Khakassia, Bashkortostán, Vladimir y Sverdlovsk Oblasts, y otras) introdujeron restricciones a la venta de bebidas alcohólicas debido a las preocupaciones sobre el posible aumento en el consumo de alcohol inducido por la pandemia asociado con otros resultados sociales y de salud adversos.
Estos diversos cambios y alteraciones en la vida causados por la crisis pandémica sugirieron una alta probabilidad de un aumento o, al menos, una desaceleración en la reducción del consumo de alcohol no registrado en Rusia en 2020, particularmente entre los sectores más desfavorecidos de la población rusa (p. ej., desempleados/con empleo irregular, pobres, sin techo, grandes bebedores). Durante la pandemia, el consumo de alcohol no registrado podría haber vuelto a ser más frecuente, al igual que lo ha sido repetidamente durante una serie de crisis socioeconómicas anteriores que han ocurrido en Rusia durante los últimos 35 años (Nemtsov, 2011; Leon y Shkolnikov, 1998; Lysova y Pridemore, 2010; OMS, 2020).

$\mathrm{El}$ alcohol no registrado en Rusia incluye varias fuentes de etanol barato, como bebidas alcohólicas no declaradas, libres de impuestos y falsificadas, alcohol casero, alcohol sustituto/no-bebida y otras fuentes. Los alcoholes no-bebida incluyen una variedad de tipos de líquidos consumidos para ingerir su contenido de alcohol: líquidos espirituosos de perfumería/cosméticos falsificados legales e ilícitas, aditivos para el baño con líquidos espirituosos, tinturas medicinales, antisépticos, etanol técnico/medicinal (Gil et al., 2018b). Con los años, estos alcoholes podían adquirirse en el comercio minorista en Rusia (Gil et al., 2009; Koshkina et al., 2013; Neufeld, Lachenmeier, Hausler y Rehm, 2016; Gil et al., 2018a) y su consumo ha sido asociado asociado con un aumento de siete veces el riesgo de muerte, espe-

Recibido: Noviembre 2020; Aceptado: Noviembre 2020.

Enviar correspondencia a: Dr. Artyom Gil. Institute for Leadership and Health Management. I.M. Sechenov First Moscow State Medical University (Sechenov University), Moscow, Russia.

E-mail: gil.artyom@gmail.com. 
cialmente entre la población en edad de trabajar (Leon et al., 2007). El principal peligro de los alcoholes no-bebida se asocia con su contenido de etanol altamente concentrado de bajo coste (hasta $95 \%$ por volumen). Su bajo coste por unidad de botella también les hace asequible para la mayoría de las personas que abusan del alcohol, incluso las más pobres.

Durante el período entre enero 2018 y septiembre 2020, realizamos un breve análisis de la disponibilidad de bebidas alcohólicas no-bebida e ilegales en cinco ciudades rusas: Odintsovo, Moscow, Izhevsk, Chelyabinsk y Petushki. En cada ciudad, los investigadores de campo visitaron hasta cinco puntos de venta y compraron muestras de alcohol no-bebida. También se compraron las bebidas alcohólicas ilícitas si se identificaron en los puntos de venta visitados.

En total, se visitaron 27 puntos de venta: farmacias, mercados, supermercados, pequeñas tiendas, quioscos, pabellones. Se compraron 126 muestras de alcohol no-bebida en estos puntos de venta, con un coste habitual de no más de 45 rublos $(\$ 0,57, € 0,49, £ 0,44)$ y contenido de $60 \%$ o más de alcohol etílico por volumen. De las 126 muestras, 59 se compraron en 2020: 35 tinturas medicinales, 9 antisépticos, 1 botella de etanol medicinal, 4 aguas de colonia con alcohol desnaturalizado y 7 con alcohol no desnaturalizado, 1 loción con alcohol no desnaturalizado, 2 desinfectantes espirituosos para la desinfección de manos. El 62,7 $\%$ de ellos se habían informado ser tipos consumidos como bebida por personas con trastornos por uso de alcohol (Bobrova et al., 2009; Neufeld et al., 2016; Gil et al., 2018a). El $57,6 \%$ y $32,2 \%$ de ellos contenían unidades más baratas de etanol ( $10 \mathrm{ml}$ de etanol puro) que el contenido del vodka ruso estándar y del vodka ilegal, respectivamente. Seis de los puntos de venta visitados que abrían 24 horas al día vendían alcohol no-bebida utilizado para beber.

El desinfectante espirituoso para la desinfección de manos comprado apareció en el mercado en los primeros meses de la pandemia de SARS-CoV-2 en 2020. Estaban a la venta 24 horas al día en las pequeñas tiendas de barrio a un bajo precio por unidad de botella (36 rublos: $\$ 0,45$, $€ 0,39, £ 0,35)$, no estaban desnaturalizados, contenían etanol altamente concentrado (95\% por volumen) con un coste unitario (10 $\mathrm{ml}$ puros de etanol) por debajo del precio estándar del vodka ruso, estaban envasados en botellas sin dispensador, no se mostraban en los escaparates y se utilizaban para beber, según las observaciones de un investigador de campo (Figura 1).

En 4 de los puntos de venta visitados en 2020 con venta de alcohol no-bebida, los investigadores de campo identificaron y compraron 9 muestras de bebidas alcohólicas ilícitas. Incluyeron vodka ilegal y coñacs, whisky y ron falsificados a la venta a precios inferiores a los precios mínimos establecidos por el estado para bebidas alcohólicas para 2020.

Los resultados de nuestro breve análisis de la disponibilidad del alcohol no registrado sugieren que permanecieron disponibles una variedad de tipos de alcohol no-bebida utilizadas para beber, además de bebidas alcohólicas ilegales, en ciudades rusas en 2020, mientras que se introdujeron al mercado durante los primeros meses de la pandemia de la COVID-19 nuevos tipos de alcohol no registrado bebibles (p. ej., desinfectantes espirituosos para la desinfección de manos). Estos alcoholes están principalmente fabricados a partir del etanol más barato disponible, que suele ser etanol farmacéutico/médico desviado del mercado legal. Es necesario en Rusia un endurecimiento de las políticas de control implementadas previamente y descritas en detalle en otras publicaciones (OMS, 2019), así como la introducción de nuevos reglamentos dirigidos a la disponibilidad y el consumo de alcohol no registrado. Endurecer el control de este alcohol puede ser particularmente importante durante la pandemia que, mediante diferentes mecanismos, como el estrés y la reducción de la asequibilidad y la dispo-

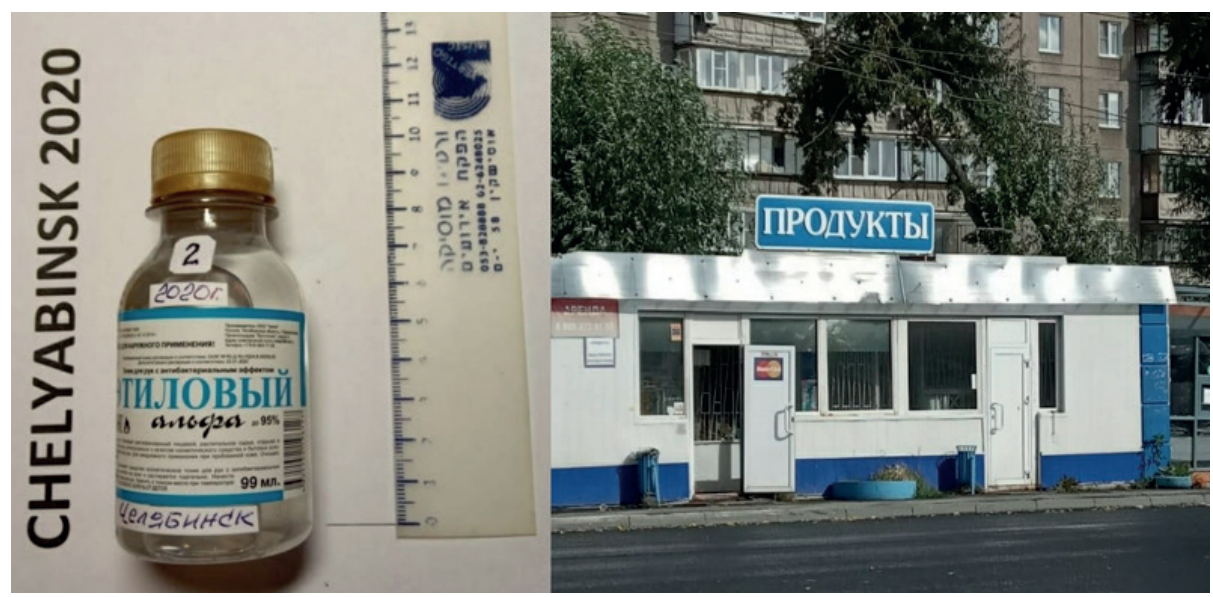

Figura 1. Desinfectante de las manos contra el SARS-CoV-2 («hand tonic Ethyl Alpha») utilizado como bebida, según las observaciones de un investigador de campo, y una pequeña tienda de barrio que lo tiene a la venta 24 horas al día. Chelyabinsk, Rusia, 2020. 
nibilidad física de bebidas alcohólicas legales, puede haber aumentado la demanda y el consumo de varios tipos de alcohol no registrado. Un control más estricto del alcohol no registrado durante la pandemia puede ser especialmente beneficioso para la prevención de la mortalidad prematura atribuible al alcohol entre los sectores más desfavorecidos social y económicamente y afectados por la crisis de la pandemia de la población en edad de trabajar, cuyas tasas de mortalidad durante varias décadas han tenido una fuerte influencia en las fluctuaciones de la mortalidad en Rusia en su conjunto.

\section{Reconocimientos}

Agradecemos a Uri Gil y Raisa Gil (Udovichenko) por identificar, comprar y fotografiar para esta publicación un desinfectante de las manos contra el SARS-CoV-2, utilizado para beber por los consumidores de alcoholes sustitutos en la ciudad de Chelyabinsk. Este estudio no recibió apoyo financiero alguno.

\section{Conflicto de intereses}

El autor declara la inexistencia de conflicto de interés.

\section{Referencias}

Artamonov, R. E. y Lavrentyev N. V. (2020). Public opinion poll of the population of the Russian Federation. Changes caused by the coronavirus epidemic and self-isolation regime. Analytical Bulletin of the National Research University "Higher School of Economics", 3, 4-24. Recuperado de https://www.hse.ru/mirror/pubs/share/368507661.pdf.

Bobrova, N., West, R., Malutina, D., Koshkina, E., Terkulov, R. y Bobak, M. (2009). Drinking alcohol surrogates among clients of an alcohol-misuser treatment clinic in Novosibirsk, Russia. Substance Use and Misuse, 44, 18211832. doi:10.3109/10826080802490717.

Gil, A., Khalfin, R., Ilchenko, I., Krinitsky, S., Kosagovskaya, I. y Fattakhova, L. (2018a). Non beverage alcohols in Russia: Were they still consumed for drinking in 20152017? Revue d'Épidémiologie et de Santé Publique, 66, 242. doi:10.1016/j.respe.2018.05.027.

Gil, A., Khalfin, R., Ilchenko, I., Savchuk, S., Kosagovskaya, I. y Madyanova, V. (2018b). Availability of nonbeverage alcohols in 50 Russian cities in 2015-2017. Revue d'Épidémiologie et de Santé Publique, 66, 356. doi:10.1016/j. respe.2018.05.325.

Gil, A., Polikina, O., Koroleva, N., McKee, M., Tomkins, S. y Leon, D. (2009). Availability and characteristics of nonbeverage alcohols sold in 17 Russian cities in 2007. Alcoholism: Clinical and Experimental Research, 33, 79-85. doi:10.1111/j.1530-0277.2008.00813.x.
Koshkina, E., Pavlovskaya, N., Vyshinski, K., Gorbachev, I., Bogdanova, N., Lenckaya, G.,... Holdin, V. (2013). Evaluation of the character and size of illegal alcohol consumption in some regions of middle Russia. Narkologia, 8, 28-36.

Lancet COVID-19 Commissioners, Task Force Chairs, and Commission Secretariat (2020). Lancet COVID-19 Commission Statement on the occasion of the 75th session of the UN General Assembly. Lancet, 396, 1102-1124. doi:10.1016/S0140-6736(20)31927-9.

Leon, D.A., Saburova, L., Tomkins, S., Andreev, E., Kiryanov, N., McKee, M.,... Shkolnikov, V.M. (2007). Hazardous alcohol drinking and premature mortality in Russia: A population based case-control study. Lancet, 369, 2001-2009. doi:10.1016/S0140-6736(07)60941-6.

Leon, D. A. y Shkolnikov, V.M. (1998). Social Stress and the Russian Mortality Crisis. JAMA, 279, 790-791. doi:10.1001/jama.279.10.790.

Lysova, A.V. y Pridemore, W.A. (2010). Dramatic problems and weak policy. Trends in alcohol consumption, harms and policy: Russia 1990-2010. Nordic Studies on Alcohol and Drugs, 27, 425-447.

Nemtsov, A.V. (2011). A Contemporary History of Alcohol in Russia. Södertörn: Södertörn University.

Neufeld, M., Lachenmeier, D., Hausler, T. y Rehm, J. (2016). Surrogate alcohol containing methanol, social deprivation and public health in Novosibirsk, Russia. International Journal of Drug Policy, 37, 107-110. doi:10.1016/j. drugpo.2016.08.001.

Organización Mundial de la Salud. (2020). COVID-19 significantly impacts health services for noncommunicable diseases. Recuperado de https://www.who.int/ news-room/detail/01-06-2020-covid-19-significantly-impacts-health-services-for-noncommunicable-diseases.

Organización Mundial de la Salud. (2019). Alcohol policy impact case study: The effects of alcohol control measures on mortality and life expectancy in the Russian Federation. Recuperado de https://www.euro.who.int/ en/publications/abstracts/alcohol-policy-impact-case-study-the-effects-of-alcohol-control-measures-on-mortality-and-life-expectancy-in-the-russian-federation-2019.

RosStat (Federal State Statistics Service of the Russian Federation). (2020). Official Statistics. Recuperado de https://eng.gks.ru/folder/11335. 
